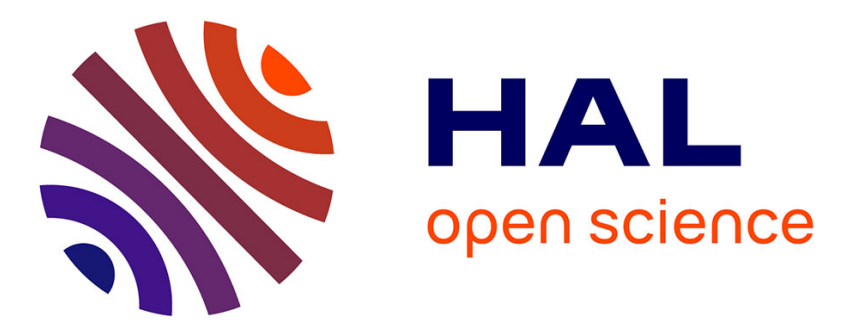

\title{
Decision Directed Channel Estimation and High I/Q Imbalance Compensation in OFDM Receivers
}

\author{
Sylvain Traverso, Myriam Ariaudo, Inbar Fijalkow, Jean-Luc Gautier, \\ Christian Lereau
}

\section{- To cite this version:}

Sylvain Traverso, Myriam Ariaudo, Inbar Fijalkow, Jean-Luc Gautier, Christian Lereau. Decision Directed Channel Estimation and High I/Q Imbalance Compensation in OFDM Receivers. IEEE Transactions on Communications, 2009, 57 (5), pp.1246 - 1249. hal-00532872

\section{HAL Id: hal-00532872 https://hal.science/hal-00532872}

Submitted on 4 Nov 2010

HAL is a multi-disciplinary open access archive for the deposit and dissemination of scientific research documents, whether they are published or not. The documents may come from teaching and research institutions in France or abroad, or from public or private research centers.
L'archive ouverte pluridisciplinaire HAL, est destinée au dépôt et à la diffusion de documents scientifiques de niveau recherche, publiés ou non, émanant des établissements d'enseignement et de recherche français ou étrangers, des laboratoires publics ou privés. 


\title{
Decision-Directed Channel Estimation and High I/Q Imbalance Compensation in OFDM Receivers
}

\author{
Sylvain Traverso, Myriam Ariaudo, Inbar Fijalkow, Member, IEEE, Jean-Luc Gautier, Senior Member, IEEE, \\ and Christian Lereau
}

\begin{abstract}
Direct-conversion architectures suffer from the mismatch between the In-phase (I) and the Quadrature-phase (Q) branches, commonly called I/Q imbalance. Even low I/Q imbalances imply poor performance of Orthogonal Frequency Division Multiplexing (OFDM) systems. In this paper, we propose a new algorithm that uses both training and data symbols in a decision-directed fashion to jointly estimate the channel and compensate for high receiver $I / Q$ imbalance. Simulation results show that our method can compensate for high $I / Q$ imbalance values and also estimate a frequency selective channel.
\end{abstract}

Index Terms-I/Q imbalance, Orthogonal Frequency Division Multiplexing (OFDM), zero-IF, equalization.

\section{INTRODUCTION}

D IRECT-CONVERSION architectures, also called zeroIF architectures, are good candidates for producing lowcomplexity OFDM receivers. The receiver I/Q imbalance resulting from imperfect matching of the analog components in the In-phase (I) and Quadrature-phase (Q) branches drastically degrades the performance of OFDM systems [1]. In order to design a high performance receiver, it is necessary to estimate and compensate for the I/Q imbalance. A very promising approach is digital compensation. The I/Q imbalance estimation problem has been addressed in the literature, and there are two main approaches. The first one is based on the received training symbols. Tubbax et al. [1] and Yu et al. [2] use the fact that the channel frequency response estimation has several sharp transitions in the presence of I/Q imbalance. Their technique estimates the I/Q imbalance when the frequency channel response on adjacent tones is highly correlated and the I/Q imbalance is low. Tarighat et al. [3] and Schuchert et al. [4] propose an estimation technique based on a leastmean-square filtering that converges slowly and requires a high number of training symbols. Tarighat et al. [3] and Windisch et al. [5] propose a technique that can estimate the I/Q imbalance by inserting null tones in the training symbols. Thus, the received training symbols are not affected by mutual interference between pairs of symmetric subcarriers. This technique gives an estimate of the I/Q imbalance using a

Paper approved by S. K. Wilson, the Editor for Multicarrier Modulation of the IEEE Communications Society. Manuscript received April 10, 2007; revised September 6, 2007, December 21, 2007, February 1, 2008, and February 14, 2008.

S. Traverso is with the Department of Embedded Digital Systems, THALES Land \& Joint Systems, Colombes, FR-92704 (e-mail: Sylvain.TRAVERSO@fr.thalesgroup.com).

M. Ariaudo, I. Fijalkow, and J.-L. Gautier are with ETIS/ENSEA - Université de Cergy - CNRS, Cergy, FR-95014 (e-mail: \{ariaudo, inbar.fijalkow, gautier\}@ensea.fr).

C. Lereau is with the Department of Unite Communication Tactique, THALES Land \& Joint Systems, Chollet, FR-49309 (e-mail: christian.LEREAU@ fr.thalesgroup.com).

Digital Object Identifier 10.1109/TCOMM.2009.05.070161 single OFDM training symbol, but it requires the modification of the training symbols. The second approach is based on data symbols only. A blind estimation is proposed by Windisch et al. [6]: it uses the fact that the transmitted subcarriers are uncorrelated. This method needs a high number of received data symbols for a good statistical estimation of the I/Q imbalance. If the number of data symbols is not sufficient, an error floor occurs even for high signal-to-noise ratios.

In this paper we are interested in the case where: (i) the adjacent subcarriers' correlation is low corresponding to a dense multipath environment; (ii) there are few training and data symbols; (iii) the I/Q imbalance can be strong; (iv) the data subcarriers carry high-order modulations and are very sensitive to I/Q imbalance. In these conditions the estimation methods presented above cannot be used. We propose to jointly compensate for high I/Q imbalance and to estimate and equalize the channel frequency response. Our method can be used for any OFDM system, and converges very quickly as it needs only few training and data symbols. It is original in the sense that it uses both the training and data received symbols in a decision-directed fashion. The remainder of the paper is organized as follows: Section II introduces the I/Q imbalance modeling in OFDM systems. Section III presents the proposed method in the case of the reception of two different training symbols. Section IV deals with the more general case of the reception of a single training symbol, and describes an iterative algorithm using both the training symbol and the data symbols. Finally Section V presents the choice of the parameters setting of our algorithm and the experimental results.

\section{I/Q IMBALANCE Modeling IN OFDM SystemS}

The I/Q imbalance is caused by a gain mismatch $g$ and a phase mismatch $\phi$. The gain mismatch is due to a difference of the overall gain between the I and Q branches. The phase mismatch is due to a non-ideal layout, i.e., the fact that the lines between the mixer and the local oscillator of the I and Q branches are not exactly of the same length. Let $K_{1} \triangleq\left(1+g e^{-j \phi}\right) / 2$ and $K_{2} \triangleq\left(1-g e^{+j \phi}\right) / 2$ be the I/Q imbalance parameters as defined in [6]. For an OFDM system impaired by the receiver I/Q imbalance, the received signal on the $k^{t h}$ subcarrier during the $n^{\text {th }}$ symbol $R_{k}(n)$ can be written as [6]:

$$
\begin{aligned}
R_{k}(n) & =T_{k}(n) H_{k} K_{1}+T_{-k}^{*}(n) H_{-k}^{*} K_{2} \\
& =T_{k}(n) \alpha_{k}+T_{-k}^{*}(n) \beta_{k}, \\
& k \in \pm\left[1 ; N_{D F T} / 2-1\right]
\end{aligned}
$$

where $\alpha_{k} \triangleq H_{k} K_{1}$ and $\beta_{k} \triangleq H_{-k}^{*} K_{2} . T_{k}(n), H_{k}$ and $N_{D F T}$ denote respectively the transmitted symbols, the channel fre- 
quency response not affected by I/Q imbalance, and the block size of the Discrete Fourier Transform. The matrix form of the received symbols defined by equation (1) is:

$$
\left[\begin{array}{c}
R_{k}(n) \\
R_{-k}^{*}(n)
\end{array}\right]=\underbrace{\left[\begin{array}{cc}
K_{1} & K_{2} \\
K_{2}^{*} & K_{1}^{*}
\end{array}\right]}_{\mathbf{A}_{1}}\left[\begin{array}{cc}
H_{k} & 0 \\
0 & H_{-k}^{*}
\end{array}\right]\left[\begin{array}{c}
T_{k}(n) \\
T_{-k}^{*}(n)
\end{array}\right] .
$$

Note that our model does not take into account the unavoidable frequency offset between the transmitter/receiver local oscillators. The estimation of the frequency offset in the presence of I/Q imbalance can be performed using the method proposed in [7] for example. Thus we assume a pre-compensation at the transmitter or an analog pre-compensation of this effect at the receiver.

\section{Two Different Long Training Symbols}

In this section, we show that when two different Long Training Symbols (LTS) are transmitted at the beginning of each packet, it is possible to estimate the I/Q imbalance and thus to jointly estimate the channel and compensate for the I/Q imbalance. The channel estimate $\left(C_{k}\right)$ is obtained by dividing the received LTS $\left(P r_{k}\right)$ by the transmitted LTS $\left(P t_{k}\right)$ that are known to the receiver. Note that $P r_{k}$ and $P t_{k}$ correspond respectively to $R_{k}$ and $T_{k}$ in the system model given by equation (1). When two different LTS are transmitted consecutively, the two channel estimations $C_{k}(1)$ and $C_{k}(2)$ affected by I/Q imbalance form a system of linear equations in two variables:

$$
\begin{aligned}
& C_{k}(1)=\frac{P r_{k}(1)}{P t_{k}(1)}=\alpha_{k}+L_{k}(1) \beta_{k} \\
& C_{k}(2)=\frac{P r_{k}(2)}{P t_{k}(2)}=\alpha_{k}+L_{k}(2) \beta_{k}
\end{aligned},
$$

where $L_{k}(1)=P t_{-k}^{*}(1) / P t_{k}(1)$ and $L_{k}(2)=$ $P t_{-k}^{*}(2) / P t_{k}(2)$ are known to the receiver. If the condition $\mathcal{C}$ defined by $L_{k}(1) \neq L_{k}(2)$ is satisfied, the system (3) can be solved. By definition $K_{1}+K_{2}^{*}=1$, and knowing $\alpha_{k}$ and $\beta_{k}$ (or their estimate), the channel frequency response is given by:

$$
H_{k}=\alpha_{k}+\beta_{-k}^{*} .
$$

Since $\alpha_{k}=K_{1} H_{k}$, equation (4) yields to the following I/Q imbalance parameters:

$$
\begin{aligned}
K_{1} & =\frac{\alpha_{k}}{\alpha_{k}+\beta_{-k}^{*}}, \\
K_{2} & =1-K_{1}^{*} .
\end{aligned}
$$

Once the I/Q parameters and the channel frequency response have been estimated, the data symbols are compensated for I/Q imbalance inverting matrix $\mathbf{A}_{1}$ in equation (2), and the channel equalization can be performed.

\section{A Single Long Training Symbol}

In many OFDM standards the same LTS is sent twice [8]. In that case the condition $\mathcal{C}$ is not satisfied, and it is not possible to apply the method described in Section III. In this Section we propose a new method for joint compensation of the I/Q imbalance and estimation of the channel frequency response using only a single LTS and data symbols. Note that if known pilots subcarriers are inserted in the data symbols satisfying the criteria $\mathcal{C}$, they can be used as a second LTS as defined in Section III. Since pilot subcarriers do not always satisfy the condition $\mathcal{C}$ and with the aim of reducing the number of pilots, in the sequel we only consider data subcarriers. The proposed algorithm is iterative. In first Subsection we present the algorithm with a single iteration, and in the second Subsection we give the general case of $i, i>1$, iterations.

\section{A. Algorithm with a single iteration}

We now present the 4 steps of our algorithm.

1) Hard decisions on $N$ received data symbols: Even if the I/Q imbalance is pronounced, the $N$ received symbols $R_{k}(n)$ equalized by the channel estimation $C_{k}$ are good approximations of $T_{k}(n)$. We denote the hard decisions of these equalized symbols as $\mathcal{D}\left[R_{k}(n) / C_{k}\right]$.

2) Estimation of the $I / Q$ imbalance parameters $K_{1}$ and $K_{2}$ : If $T_{k}(n)$ and $T_{-k}^{*}(n)$ are replaced by the previous hard decisions in equation (1), we obtain an equation equivalent to the second received LTS, as described in Section III. Let $\zeta$ be a set of all subcarriers satisfying $\mathcal{D}\left[R_{k}(n) / C_{k}\right] L_{k} \neq$ $\mathcal{D}\left[R_{-k}^{*}(n) / C_{-k}^{*}\right]$. For each subcarrier $k$ in $\zeta$, the estimation of $\alpha_{k}$ and $\beta_{k}$ is possible. Since $K_{1}$ is the same for all the subcarriers, and since we use $N$ data symbols, it is possible to average the estimates over time and frequency:

$$
\widehat{K}_{1}=\frac{1}{\operatorname{card}(\zeta)} \sum_{k \in \zeta} \frac{\widehat{\alpha}_{k}}{\widehat{\alpha}_{k}+\widehat{\beta}_{-k}^{*}} .
$$

$\widehat{K}_{2}$ is obtained by $\widehat{K}_{2}=1-\widehat{K}_{1}^{*}$.

3) I/Q imbalance compensation of data symbols: Once $K_{1}$ and $K_{2}$ have been estimated, the data symbols are compensated for the I/Q imbalance by inverting the matrix $\mathbf{A}_{1}$ given in equation (2). The data symbols compensated for the I/Q imbalance are:

$$
\widehat{D}_{k}(n)=\frac{\widehat{K}_{1}^{*} R_{k}(n)-\widehat{K}_{2} R_{-k}^{*}(n)}{\left|\widehat{K}_{1}\right|^{2}-\left|\widehat{K}_{2}\right|^{2}} .
$$

4) I/Q imbalance compensation of the channel estimation: The estimates $\widehat{H}_{k}$ deduced from $\widehat{H}_{k}=\widehat{\alpha}_{k}+\widehat{\beta}_{-k}^{*}$ are not reliable for two reasons. The first one is that for each received data symbol, $\widehat{H}_{k}$ is not available when $k$ does not belong to $\zeta$. Secondly, $\widehat{H}_{k}$ is severely corrupted by hard decision errors because there is no averaging over the frequency range. Since the estimations of the I/Q imbalance parameters are reliable, the I/Q imbalance compensation of the rough channel estimation $C_{k}$ gives a reliable estimation of $H_{k}$ in a similar way as in the step 3 :

$$
\widehat{H}_{k}=\frac{\widehat{K}_{1}^{*} C_{k}-L_{k} \widehat{K}_{2} C_{-k}^{*}}{\left|\widehat{K}_{1}\right|^{2}-\left|\widehat{K}_{2}\right|^{2}}
$$

\section{B. The algorithm with $i$ iterations:}

The matrix form of the data symbols compensated for I/Q imbalance after the first iteration can be written as follows:

$$
\left[\begin{array}{c}
\widehat{D}_{1 k}(n) \\
{\widehat{D_{1-k}}}^{*}(n)
\end{array}\right]=\underbrace{\widehat{\mathbf{A}_{1}}{ }^{-1} \mathbf{A}_{1}}_{\mathbf{A}_{2}}\left[\begin{array}{cc}
H_{k} & 0 \\
0 & H_{-k}^{*}
\end{array}\right]\left[\begin{array}{c}
T_{k} \\
T_{-k}^{*}
\end{array}\right],
$$


where $\widehat{\mathbf{A}_{1}}$ and $\mathbf{A}_{2}$ correspond respectively to the estimationcompensation matrix of the I/Q imbalance, and to the remaining I/Q imbalance matrix after the first iteration. Simple algebra leads to $\mathbf{A}_{2}$ in the following form:

$$
\begin{gathered}
\mathbf{A}_{2}=\left[\begin{array}{ll}
K_{11} & K_{21} \\
K_{21}^{*} & K_{11}^{*}
\end{array}\right], \\
K_{11}=\frac{\widehat{K}_{1}^{*} K_{1}-\widehat{K}_{2} K_{2}^{*}}{\left|\widehat{K}_{1}\right|^{2}-\left|\widehat{K}_{2}\right|^{2}}, K_{21}=\frac{\widehat{K}_{1}^{*} K_{2}-\widehat{K}_{2} K_{1}^{*}}{\left|\widehat{K}_{1}\right|^{2}-\left|\widehat{K}_{2}\right|^{2}} .
\end{gathered}
$$

If the estimation of the I/Q imbalance parameters obtained after the first iteration was perfect, then $\mathbf{A}_{2}$ would be the identity matrix. In this case $\widehat{D_{1 k}}(n)=H_{k} T_{k}(n)$, and the I/Q imbalance would be perfectly compensated. However, in the presence of hard decision errors, the algorithm estimates imperfect I/Q imbalance parameters. From $K_{1}+K_{2}^{*}=1$ and $\widehat{K}_{1}+\widehat{K}_{2}^{*}=1$, it follows after simple algebra that $K_{11}+K_{21}^{*}=1$, regardless of the values of $K_{1}, K_{2}$ and their estimates. Therefore equation (10) is similar to equation (2). It is possible to reiterate the estimation of the remaining I/Q imbalance parameters returning to the step 1 of the core algorithm. The data symbols and the channel estimation necessary in step 1 at the iteration $i$ are those obtained in the iteration $i-1$. Thus, the matrix $\mathbf{A}_{i+1}$ after the $i^{t h}$ iteration is:

$$
\mathbf{A}_{i+1}=\widehat{\mathbf{A}}_{i}^{-1}{\widehat{\mathbf{A}_{i-1}}}^{-1} \cdots \widehat{\mathbf{A}}_{2}^{-1} \widehat{\mathbf{A}}_{1}^{-1} \mathbf{A}_{1}
$$

The experimental results evidence that $\mathbf{A}_{i+1}$ tends to the identity matrix when there is no noise and $i$ goes to infinity. Due to the space limitations, details of the iterative compensation of the channel estimation are not given, but it can be easily checked that it leads to a similar matrix as equation (11).

\section{Simulation Results}

In this section, we demonstrate the capabilities of the proposed I/Q imbalance compensation and channel estimation algorithm in the context of the 802.11a Standard [8]. The data subcarriers are modulated by a 64-QAM (Quadrature Amplitude Modulation). An independent channel realization of the Channel C [9], which is considered as a dense multipath environment, is drawn for each transmitted packet. The simulation results are depicted by averaging curves over 100 received packets. First we give the way that the parameters of the algorithm are chosen, then we analyse the performance of the algorithm, and finally we test its robustness over an extremely wide range of I/Q imbalance.

\section{A. Parameters}

The algorithm requires two parameters: the number of data symbols $N$, and the number of iterations $i$. A good criterion to choose these parameters is the conversion gain, defined as the power of the undesired complex down-conversion divided by the power of the desired complex down-conversion [6]. The conversion gain of the compensated signal after the $i^{\text {th }}$ iteration is defined by:

$$
G C_{\text {comp }}=\left|\frac{K_{2 i}}{K_{1 i}}\right|^{2}
$$

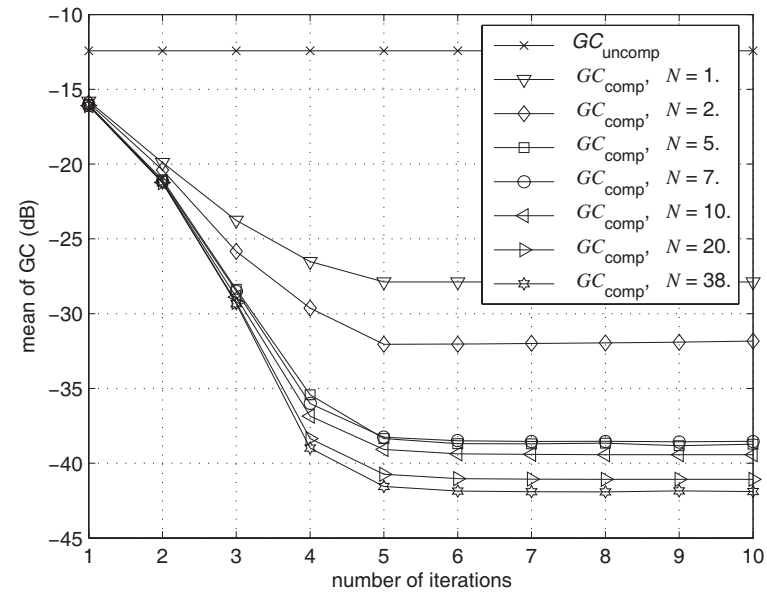

Fig. 1. The mean of conversion gains versus the number of iterations for various values $N$ of hard decisions data OFDM symbols, 64-QAM, gain mismatch $g=1.5$, phase mismatch $\phi=15^{\circ}$, Channel $\mathrm{C}$, and $E_{b} / N_{0}=25$ dB.

where $K_{1 i}$ and $K_{2 i}$ denote the remaining I/Q imbalance parameters after the $i^{t h}$ iteration. We restrict the packet error rate so that it does not exceed $10 \%$. The corresponding uncoded target BER is $10^{-2}$ and is reached for the energy per bit to noise ratio $\left(E_{b} / N_{0}\right)$ equal to $25 \mathrm{~dB}$ in the case of no I/Q imbalance (Fig. 2). Thus we propose to define the optimal number of data symbols $N$ and iterations $i$ required in order to minimize the conversion gain of the compensated signal for $E_{b} / N_{0}=25 \mathrm{~dB}$. We set the maximum tolerated I/Q imbalance to $g=1.5$ and $\phi=15^{\circ}$. Fig. 1 gives the average of $G C_{\text {comp }}$ versus the number of iterations for various values of $N$ for $g=1.5, \phi=15^{\circ}$ and $E_{b} / N_{0}=25 \mathrm{~dB}$. For reference, we define the conversion gain of the uncompensated

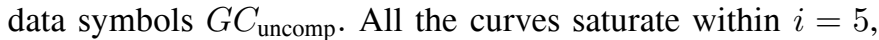
and the error floor is fixed by the value of $N$. Moreover, it appears that for $N \geq 5$, there is no significant improvement of the $G C_{\text {comp }}$ curves. The best trade off between complexity and performance is also obtained for $i=5$ and $N=5$.

\section{B. Performance}

We propose to compare the performance of the proposed algorithm to the performance in the case of no I/Q imbalance and to the performance in the case of I/Q imbalance but without any compensation scheme. Fig. 2 gives the uncoded BER curves versus $E_{b} / N_{0}$ for different values of I/Q imbalance. Even for small I/Q imbalance, the degradation due to I/Q imbalance is significant without any correction: BER curves saturate for small $E_{b} / N_{0}$ values and do not reach the target uncoded BER of $10^{-2}$. Fig. 2 shows that the performance of the proposed algorithm are very close to the BER curve without I/Q imbalance even for high I/Q imbalance.

\section{Operating range}

We now test the proposed algorithm over an extremely wide range of $\mathrm{I} / \mathrm{Q}$ imbalance, i.e. until $g=2$ and $\phi=30^{\circ}$. The parameters of the algorithm are set to $N=10$ and $i=10$ in a similar way as in Subsection V-A. A good criterion for measuring the performance of the proposed scheme is the 


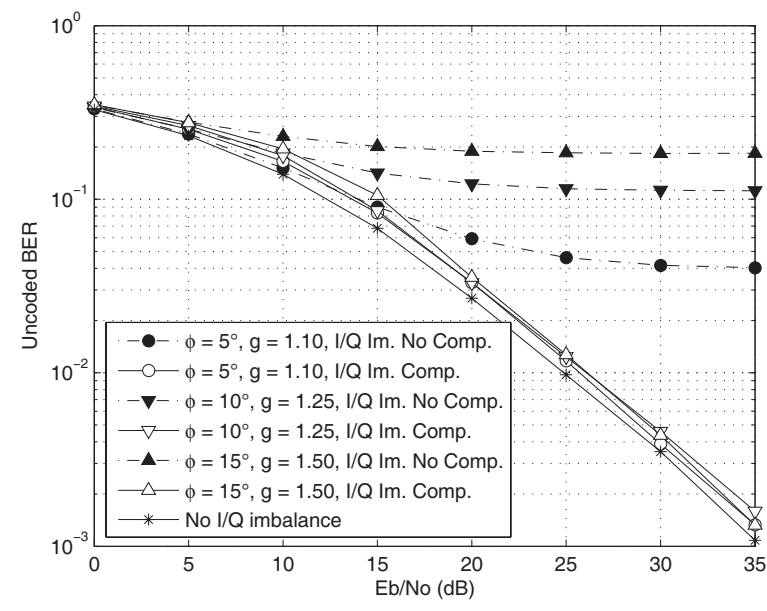

Fig. 2. The Channel $\mathrm{C}$ uncoded BER performance for the receiver with no compensation and for the receiver using the proposed algorithm. The simulations are performed for three different I/Q imbalance. "I/Q Imbalance No Comp." refers to a receiver with I/Q imbalance and no compensation scheme, "I/Q Imbalance Comp." refers to the proposed estimation and compensation scheme for a receiver with $\mathrm{I} / \mathrm{Q}$ imbalance.

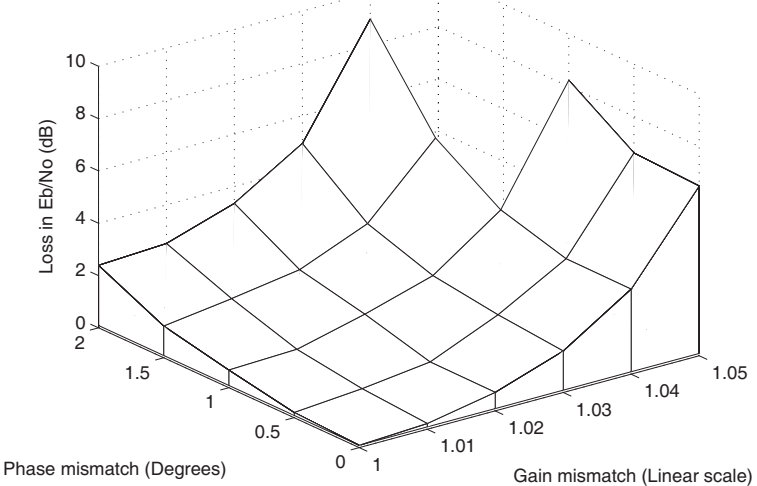

Fig. 3. Loss in $E_{b} / N_{0}$ as a function of gain and phase mismatch for a fixed uncoded BER of $10^{-2}$ when no compensation scheme is used and in the case of Channel C.

$E_{b} / N_{0}$ gap between the uncoded BER curves of a receiver with no I/Q imbalance and a receiver with I/Q imbalance. Figs. 3 and 4 give respectively the loss in $E_{b} / N_{0}$ as a function of the gain and phase mismatches for a fixed uncoded BER of $10^{-2}$ when the I/Q imbalance is not corrected, and when the I/Q imbalance is estimated and compensated for with the proposed algorithm. Note that the loss in $E_{b} / N_{0}$ is not given when that the uncoded BER curves saturate to a value higher than the desired $10^{-2}$. We note that it is not possible to reach the target BER bellow $10^{-2}$ even for small values of mismatch when the I/Q imbalance is not compensated for. The performance degradation attained with our algorithm is below $1.5 \mathrm{~dB}$ even for extremely high I/Q imbalance.

\section{CONCLUSION}

Due to low-cost requirements for consumer product applications, the perfect match between I and Q branches at the receiver is not possible in the analog domain. The I/Q imbalance affects the received training symbols, as well as the data symbols implying that: (i) the channel estimation and the data symbols are erroneous, and (ii) the equalization is

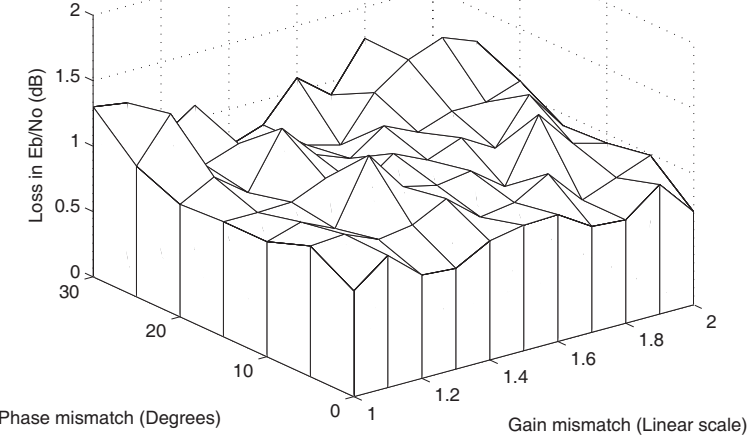

Fig. 4. Loss in $E_{b} / N_{0}$ as a function of gain and phase mismatch for a fixed uncoded BER of $10^{-2}$ for a receiver with the proposed algorithm and in the case of Channel C. The loss in $E_{b} / N_{0}$ compared to a transmission with no $\mathrm{I} / \mathrm{Q}$ imbalance is less than $1.5 \mathrm{~dB}$, even for very strong I/Q imbalance such as $g=2$ and $\phi=30^{\circ}$.

performed coarsely. These problems lead to a saturation of the BER curves even for small I/Q imbalance in a frequency selective environment. We propose an iterative algorithm for the joint channel estimation and I/Q imbalance compensation in OFDM receivers. This algorithm uses both the training and data received OFDM symbols in a decision-directed fashion. Even with no change of the pilots symbols, it leads to significantly improved performance, approaching the performance of a receiver not affected by I/Q imbalance.

\section{ACKNOWLEDGEMENT}

The authors would like to thank Auguste Venkiah for helpful discussions. They also wish to thank the anonymous reviewers for their helpful comments which improved the quality of this paper.

\section{REFERENCES}

[1] J. Tubbax, B. Côme, L. van der Perre, L. Deneire, S. Donnay, and M. Engels, "Compensation of IQ imbalance in OFDM systems," in Proc. IEEE International Conf. on Commun. (ICC), May 2003, pp. 3403-3407.

[2] J.-Y. Yu, M.-F. Sun, T.-Y. Hsu, and C.-Y. Lee, "A novel technique for I/Q imbalance and CFO compensation in OFDM systems," in Proc. IEEE International Symposium on Circuits and Systems (ISCS), May 2005, pp. 6030-6033.

[3] A. Tarighat, R. Bagheri, and A. H. Sayed, "Compensation schemes and performance analysis of IQ imbalances in OFDM receivers," IEEE Trans. Signal Processing, vol. 53, no. 8, pp. 3257-3268, Aug. 2005.

[4] A. Schuchert, R. Hasholzner, and P. Antoine, "A novel IQ imbalance compensation scheme for the reception of OFDM signals," IEEE Trans. Consumer Electron., vol. 47, no. 3, pp. 313-318, Aug. 2001.

[5] M. Windisch and G. Fettweis, "Preamble design for an efficient I/Q imbalance compensation in OFDM direct-conversion receivers," in Proc. 10th International OFDM Workshop, Sept. 2005.

[6] — , "Standard-independent I/Q imbalance compensation in OFDM direct-conversion receivers," in Proc. 9th International OFDM Workshop, Sept. 2004, pp. 57-61.

[7] F. Yan, W.-P. Zhu, and M. O. Ahmad, "Carrier frequency offset estimation and I/Q imbalance compensation for OFDM systems," EURASIP J. Advances in Signal Processing, vol. 2007, pp. 453-464, 2007.

[8] Wireless LAN Medium Access Control (MAC) and PHYsical Layer (PHY) Specifications: High-Speed Physical Layer in the $5 \mathrm{GHz}$ Band, IEEE standard 802.11a-1999 - part 11 Std. [Online]. Available: http://standards.ieee.org/getieee802/download/802.11-1999.pdf

[9] Channel Models for HiperLAN2 in Different Indoor Scenarios, European Telecommunication Standard Institute (ETSI) Broadband Radio Access Networks (BRAN) Std., Mar. 1998. 